\title{
Decimal Numbers: A Potential Bridge between Rational Numbers Representations
}

\author{
Cristina Morais \\ Lurdes Serrazina \\ UIDEF, Instituto de Educação, Universidade de Lisboa, Portugal
}

In this paper, we focus on the transformations among rational number representations made by students when working with decimal numbers. A teaching experiment was designed and carried out with the same class of 25 students, in Grade 3 and 4. We present evidence of two moments of the teaching experiment: the first is an excerpt of a whole-class discussion in the third grade, and the second is individual interviews with 4 students in the fourth grade. The results reveal that students are able to transform representations into equivalent forms that they perceive as more efficient. They are also able to regroup a decimal number by coordinating fraction and percentage knowledge as well as flexibly shifting the unit. Both strategies appear to empower the development of rational number comprehension.

Keywords: Decimal numbers, transformations, representations, rational numbers, early years.

A large body of work about learning and teaching of rational numbers shows it to be a complex and challenging topic. Studies related to decimal numbers ${ }^{1}$ focus on this symbolic representation specificities and stress the difficulties that arise when dealing and operating with this number representation. Forms of extending whole number knowledge that can lead to errors (such as perceiving 0.15 as higher than 0.3 because 15 is higher than 3 ), and errors specific to decimal numbers have been wide reported (e.g., Baturo, 2000; Durkin \& Rittle-Johnson, 2015; Resnick, Nesher, Leonard, Magone, Omanson \& Peled, 1989; Steinle \& Stacey, 2003). Additional studies suggest that such difficulties may remain until adulthood (e.g., Vamvakoussi, Van Dooren, \& Verschaffel, 2012) due in part to the demanding conceptual understanding needed regarding this representation of rational numbers.

The didactical approach is crucial to overcome these difficulties, focusing on conceptual understanding of decimal numbers, which provides meaning for procedural understanding. Furthermore, decimal numbers should not be approached in isolation, but rather in connection to other mathematical

\footnotetext{
${ }^{1}$ The term "decimal numbers" is used to identify positive rational numbers written according to decimal system notation, using the decimal point.
} 
constructs, such as other rational number representations. As Lachance and Confrey (2002) emphasize, to “. . . truly understand decimals, we must understand the connections between decimal notation and place value, fractions, and percents" (p. 506).

We believe that a sound understanding of rational numbers in its decimal representation involves not only being able to alternate between rational numbers' representations, such as $0.06=6 / 100$, but also being able to transform decimal numbers in various ways according to the situation and knowledge. For example, in the same way that students can flexibly transform whole numbers such as 14 into $10+4,7+7$ or other forms according to the situation, the development of this flexibility should also be promoted with decimal numbers (e.g., $0.55=0.5+0.05,0.25+0.3, \ldots$ ). Therefore, in this paper, we sought to answer the following question: What type of transformations among rational number representations do students make when working with decimal numbers?

\section{Understanding Decimal Numbers}

Rational numbers encompass multiple mathematical ideas, among them the idea that multiple representations can express the same value. While also true with whole numbers, rational numbers entail different forms of symbolic representations found in decimals, fractions, and percentages. Representations have an important role in the development of rational number understanding, as they appear to be related to (i) flexibility with transformations between rational number representations, (ii) flexibility with transformations within a representation, and (iii) progressive independence from concrete embodiments of rational numbers (Post, Cramer, Behr, Lesh, \& Harel, 1993).

Transformations among and within representations allow students not only to learn how to alternate between equivalents forms but also to select and use a more efficient representation to solve problems or express quantities (National Council of Teachers of Mathematics [NCTM], 2000). Also, the use of alternative forms of the same underlying concept promotes students' ability to integrate these representations as part of the same number domain (Owens \& Super, 1993). Goldin (2003) also stresses that mathematical concepts are more powerful when one develops appropriate representations for and connections among them. Moreover, a representation by itself has limited meaning, and that is through the connections one establish that this meaning can be built on (Goldin, 2003). This meaning also develops through a continuous interaction between the student's own understanding and the representations presented to him as symbolic representations.

Hence, flexibility in transforming representations requires conceptual understanding of the underlying ideas of and relationships among the representations. As Lachance and Confrey (2002) stress, for a concept to be 




\section{Findings}

We focused on two moments of the teaching experiment: an excerpt from a whole-class discussion that took place in the middle of Grade 3 and the individual interview at the end of Grade 4 with the four students previously selected. All students' names presented in the analysis' section are fictitious.

Prior to the research, students had formally been introduced to rational numbers, only in fraction representation. Fractions had been first approached with a part-whole meaning, with continuous units, and were after used as operators of discrete units. No prior teaching had been done with decimals or percent.

\section{Establishing Connections Among Representations - Whole-class Discussion (Grade 3)}

In previous research lessons, students had explored decimal numbers connected with water bottle capacities and to the number line graduated in a tenths scale. The hundredths had been addressed as the unit used when one tenth needed to be further divided into ten equal parts. The hundredths were used as half of a tenth, as in $0.15,0.25,0.35 \ldots$ Students' previous knowledge related to whole numbers, fractions, decimals or percent was highly valued, and students frequently called upon their informal knowledge when solving the tasks.

In this whole-class discussion, the students were exploring two representations, a 10 grid and a 100 grid (Figure 1). These representations were projected on the TV screen in the classroom. In the task, each grid represented a towel that was going to be painted, so along the discussion, these representations were sometimes addressed as "towels".

Figure 1. Representations of the 10 grid and 100 grid.

After exploring how and into how many parts each grid was divided, the teacher asked the students what represented each bar in the 10 grid and each square in the 100 grid. This question started a discussion about different ways to represent one tenth and one hundredth. Starting with one tenth, the teacher asked students to write down on the whiteboard representations that could represent it. 
Forms of symbolic representations as the fraction $1 / 10$ and the decimal number 0.1 were written on the whiteboard; all students agreed with these forms, as they were already used in the previous lesson. Then, one student, Tomás, wrote " $10 \%$ " and explained why:

Tomás: Ten percent.

Researcher: Is it the same as one tenth?

Tomás: Yes! Because the ten percent it's the tenth part of one hundred, and one hundred is the unit.

Tomás identified the unit and regrouped one hundred percent to one unit. He also related ten of those parts with ten percent or one tenth. However, this wasn't clear for all the students. One classmate, Jorge, explained why he didn't agree:

Jorge: I don't think so because the ten percent... I would consider it more like one percent, considering that the ten percent was... like the limit!

Researcher: But what is the limit? If I want to have the whole, the whole towel, how much will I have, in percentage?

$\cdots$

Jorge: In the red towel (100 grid), I don't consider it one tenth because one tenth of that towel (100 grid) was one row (does a vertical gesture with the hand, pointing to one column) ... And the ten percent would be all the columns painted.

The student established ten percent as the unit, perhaps driven by the fact that the 10 grid was divided into ten parts or because he was seeing the ten rows in the 100 grid, so $10 \%$ would represent the unit. It could also be due to the fact that 0.1 seems to be, misleading, transformed into $1 \%$ instead of $10 \%$.

The researcher then focused on the percentage that represents the unit, comparing it to the percentage value that represents a fully charged battery for mobile phones or tablets. Jorge smiled and recognized that the whole is represented by $100 \%$. Tomás, who had written $10 \%$, added "If it was one percent, it was a hundredth".

Tomás transformed the percentage into decimal representation, and his statement was then connected to the 100 grid representation, relating one small square of the 100 grid with 0.01 and $1 \%$. This way, the transformations among representations were intended to be visually clear for all students.

Other representations for one tenth used by students were drawn on the whiteboard: a rectangular model, with one of the ten parts colored, and a number line.

One of the representations written by one student was " 0.05 and 0.05 " and he explained:

Frederico: It is to join the two (makes a line connecting both decimal numbers).

Researcher: Ok, is to join. And how can we read that?

Frederico: Zero comma zero five plus... and... zero comma zero five. 


he didn't feel the need to say the zero seems to reveal understanding of the decimal number system, particularly about the role of the zero when placed in the rightmost place in the decimal part of the number.

Alfonso was also asked if 0.205 wouldn't be the highest one. With confidence, he said immediately "no" and explained:

Afonso: No, because if we take one zero... In this case, it's not a zero.

Here it's the five; we see how it is in the hundredths; it is zero comma twenty. That is, that the hundredths are not always greater.

Researcher: The hundredths are not always greater?

Afonso: The thousandths! The thousandths are not always greater. For example, if here it was two hundred and fifty (instead of 0.205), this [0.250] would be equal to this one [1/4]. But now, if it was two hundred and fifty five, this [1/4] was smaller.

Afonso started by pointing out what could seem to be procedural knowledge, indicating that one could take the five of 0.205 and think about 0.20 . However, this procedure seemed to be linked to conceptual knowledge about the decimal number system. Afonso addressed the question that the magnitude of a decimal number does not depend on the number of digits of the decimal part, and that decimal numbers cannot be equated to whole numbers. $\mathrm{He}$ also provided a counterexample, comparing 0.255 to 0.25 (1/4).

Afonso organized the numbers as: 1/4, 0.205, 20\%, 0.025, and 0.002 . He seemed to transform the different representations into decimals, using this representation to compare numbers. When he did it, he did not compare the representations as whole numbers. Instead, he revealed an understanding of the positional value of the digits that formed each decimal number.

Rute seemed to organize the numbers quickly, easily moving between symbolic representations:

Rute: Zero comma twenty five (1/4), zero comma twenty (20\%), one quarter $(1 / 4) \ldots$ This would be one fifth $(20 \%) \ldots$

She read both $1 / 4$ and $20 \%$ as decimals, and then she easily converted $20 \%$ to one fifth. Rute quickly organized the tags into the order: $1 / 4,20 \%$, 0.205, 0.025 and 0.002 and explained:

Rute: One quarter (1/4) equals twenty-five, for example. Twenty percent, hum... is twenty percent; it's one fifth. Zero comma two hundred and five, it's like also one fifth only with five more thousandths. And this [0.025] would also be one quarter of... one quarter of one tenth...? And this [0.002] would be one fifth of one hundredth (shrugged her shoulders and smiles).

Again, Rute exhibited flexibility among symbolic representations. She was able to regroup 0.205 as one fifth and five thousandths. This regrouping is different than regrouping 0.205 as, for example, 0.20 and 0.005 . She regrouped the value considering different representations, in this case, fractions and decimals. 

had thousandths, and thousandths are smaller than hundredths. Even though Bárbara could transform 20\% into hundredths in order to compare both representations, she revealed an understanding common in an early stage of learning decimal numbers (Durkin \& Rittle-Johnson, 2015; Resnick et al., 1989). We consider it an indicator of development of this student's comprehension of decimal numbers, that Bárbara relates each decimal part to the reference unit, perceiving that each part will be smaller if the unit is divided into more parts. In contrast, Afonso pointed to the fact that the magnitude of a decimal number doesn't depend on the number of its digits and provided the example that 0.255 is greater than 0.25 .

The four students interviewed seemed to be able to flexibly move among decimal numbers, fractions, and percent choosing the representation that each one felt to be more efficient in comparing the numbers. The transformations made by these four students seem to be conceptually grounded, as no procedures were followed.

One type of transformation had not been anticipated in our research, but we found it to be particularly powerful. Recalling Frederico's strategy in the first episode, he composed 0.1 as $0.05+0.05$ reading it as "half tenth plus half tenth", which seems to reveal an understanding of the decimal notation connected to a part-whole meaning. The student shifted between units, considering not 1 as the unit, but 0.1 as the unit.

Similarly, in the second episode, Rute and Dinis promptly regrouped 0.025 into $1 / 4$ of 0.1 and two percent and a half, respectively. This type of transformation seems to be significantly different than transforming, for example, 0.025 into $25 / 1000$, in which the unit remains the same. The type of transformation implies a unit shift (1 to 0.1 in Rute's case and 100\% in Dinis's transformation), and being flexible with the unit concept is central in dealing with rational numbers (Post et al., 1993).

Related to this type of transformation is the regrouping of 0.205 as $1 / 5$ and 5 thousandths made by Rute. In our perspective, this composition is related to the regrouping strategy within the reunitising identified by Baturo (2000). Although this author elaborates on this strategy only with decimal numbers, it seems to us that the regrouping strategy can also be understood as implying transformations into other forms of symbolic representations, such as percentage or fractions.

The way this strategy is used provides important evidence that helps teachers and researchers to infer about the meanings students are developing and how they are connecting decimal, fraction, and percentage symbolic representations. The fact that students relate these representations in such a way seems to contribute to what Goldin (2003) defines as effective mathematical thinking, which includes an understanding of the connections among different representations of the same concept.

The ability to transform decimal numbers into equivalent forms, such as $0.36=36 / 100=36 \%$, is undeniably important. Moreover, transforming decimal 

